\title{
Generalizations of poly-Bernoulli and poly-Cauchy numbers
}

\author{
Mehmet Cenkci $^{1}$ • Paul Thomas Young ${ }^{2}$
}

Received: 8 February 2015 / Revised: 24 July 2015 / Accepted: 3 August 2015 /

Published online: 1 September 2015

(C) Springer International Publishing AG 2015

\begin{abstract}
In this paper we consider some generalizations of poly-Bernoulli and polyCauchy numbers. The first is by means of the Hurwitz-Lerch zeta function. The second generalization is via weighted Stirling numbers. The third one is given with the help of degenerate Stirling numbers. All these generalizations lead to symmetries between various types of Stirling numbers, and enable us to investigate and expand algebraic properties of poly-Bernoulli and poly-Cauchy numbers. We also combine these generalizations and derive numerous combinatorial and arithmetical identities.
\end{abstract}

Keywords Poly-Bernoulli · Poly-Cauchy numbers · Weighted Stirling numbers · Degenerate Stirling numbers

Mathematics Subject Classification $11 \mathrm{~B} 68 \cdot 11 \mathrm{~B} 73 \cdot 11 \mathrm{~B} 75$

M. Cenkci was supported by Akdeniz University Scientific Research Projects Unit and the Scientific and Technological Research Council of Turkey (TUBITAK).

$\triangle$ Mehmet Cenkci

cenkci@akdeniz.edu.tr

Paul Thomas Young

paul@math.cofc.edu

1 Department of Mathematics, Akdeniz University, 07058 Antalya, Turkey

2 Department of Mathematics, College of Charleston, 66 George St., Charleston, SC 29424, USA 


\section{Introduction}

The Bernoulli numbers, $B_{n}, n \in \mathbb{Z}, n \geqslant 0$, defined by the generating function

$$
\frac{t}{\mathrm{e}^{t}-1}=\sum_{n=0}^{\infty} B_{n} \frac{t^{n}}{n !}
$$

play important roles in many applications in number theory, combinatorics, numerical analysis and other branches of mathematics. The first few Bernoulli numbers are $B_{0}=1, B_{1}=-1 / 2, B_{2}=1 / 6$, and $B_{n}=0$ for all odd $n \geqslant 3$.

Bernoulli numbers have several relations with combinatorial numbers. For example, we have

$$
B_{n}=(-1)^{n} \sum_{m=0}^{n} S_{2}(n, m) \frac{(-1)^{m} m !}{m+1},
$$

where $S_{2}(n, m)$ denotes the Stirling numbers of the second kind which are defined as

$$
\frac{\left(\mathrm{e}^{t}-1\right)^{m}}{m !}=\sum_{n=m}^{\infty} S_{2}(n, m) \frac{t^{n}}{n !} .
$$

These numbers are also determined by

$$
S_{2}(n, m)=\frac{1}{m !} \sum_{j=0}^{m}(-1)^{j}\left(\begin{array}{c}
m \\
j
\end{array}\right)(m-j)^{n},
$$

and $S_{2}(n, m)=0$ for $n<m$ (see [13] for other relations and properties).

There are numerous generalizations of Bernoulli numbers, including the polyBernoulli numbers defined by Kaneko [19] in 1997 as

$$
\frac{\mathrm{Li}_{k}\left(1-\mathrm{e}^{-t}\right)}{1-\mathrm{e}^{-t}}=\sum_{n=0}^{\infty} B_{n}^{(k)} \frac{t^{n}}{n !}
$$

where

$$
\operatorname{Li}_{k}(z)=\sum_{n=1}^{\infty} \frac{z^{n}}{n^{k}}
$$

is the $k$ th polylogarithm function. When $k=1$ we have $B_{n}^{(1)}=B_{n}$, the classical Bernoulli number, except that $B_{1}^{(1)}=-B_{1}$ when $n=1$. The generating function of the poly-Bernoulli numbers may also be written in terms of iterated integrals as 


$$
\mathrm{e}^{t} \frac{1}{\mathrm{e}^{t}-1} \underbrace{\int_{0}^{t} \frac{1}{\mathrm{e}^{u}-1} \int_{0}^{t} \frac{1}{\mathrm{e}^{u}-1} \cdots \int_{0}^{t} \frac{u}{\mathrm{e}^{u}-1}}_{(k-1) \text {-times }} \underbrace{\mathrm{d} u \mathrm{~d} u \cdots \mathrm{d} u}_{(k-1) \text {-times }}=\sum_{n=0}^{\infty} B_{n}^{(k)} \frac{t^{n}}{n !},
$$

and a combinatorial formula for $B_{n}^{(k)}$ is

$$
B_{n}^{(k)}=(-1)^{n} \sum_{m=0}^{n} \frac{(-1)^{m} m ! S_{2}(n, m)}{(m+1)^{k}} .
$$

Thus putting $k=1$ gives (1). The poly-Bernoulli numbers are related to multiple zeta values (see [2,3]), and for comprehensive information we refer to [1, Chapter 14].

The Cauchy numbers are denoted by $c_{n}$ and defined as the definite integral of the falling factorial

$$
c_{n}=\int_{0}^{1} t(t-1) \cdots(t-n+1) \mathrm{d} t=n ! \int_{0}^{1}\left(\begin{array}{l}
t \\
n
\end{array}\right) \mathrm{d} t
$$

(see [27]). The generating function of Cauchy numbers is given by

$$
\frac{t}{\log (1+t)}=\sum_{n=0}^{\infty} c_{n} \frac{t^{n}}{n !}
$$

Cauchy numbers share a particular relationship with Bernoulli numbers of the second kind $b_{n}$, in that $c_{n}=n ! b_{n}$ (for detailed information on $b_{n}$ we refer to $[6,17,28]$ ). Cauchy numbers satisfy the combinatorial formula

$$
c_{n}=(-1)^{n} \sum_{m=0}^{n} \frac{(-1)^{m} S_{1}(n, m)}{m+1},
$$

where $S_{1}(n, m)$ are the (unsigned) Stirling numbers of the first kind which are defined by

$$
\frac{[\log (1+t)]^{m}}{m !}=\sum_{n=m}^{\infty}(-1)^{n-m} S_{1}(n, m) \frac{t^{n}}{n !}
$$

These numbers also arise as the coefficients of the rising factorial

$$
t(t+1) \cdots(t+n-1)=\sum_{m=0}^{n} S_{1}(n, m) t^{m}
$$

and $S_{1}(n, m)=0$ if $n<m$. 
The definite integral defining the Cauchy numbers and the iterated integral expression for poly-Bernoulli numbers motivate a definition of poly-Cauchy numbers as

$$
c_{n}^{(k)}=n ! \underbrace{\int_{0}^{1} \cdots \int_{0}^{1}}_{k \text {-times }}\left(\begin{array}{c}
t_{1} t_{2} \cdots t_{k} \\
n
\end{array}\right) \mathrm{d} t_{1} \mathrm{~d} t_{2} \cdots \mathrm{d} t_{k},
$$

(see [22]). For $k=1, c_{n}^{(1)}=c_{n}$ are the Cauchy numbers. The poly-Cauchy numbers and the Stirling numbers of the first kind share a particular relationship in that

$$
c_{n}^{(k)}=(-1)^{n} \sum_{m=0}^{n} \frac{(-1)^{m} S_{1}(n, m)}{(m+1)^{k}} .
$$

To give a generating function for poly-Cauchy numbers, let $\operatorname{Lif}_{k}(z)$ be the polylogarithm factorial function defined by

$$
\operatorname{Lif}_{k}(z)=\sum_{m=0}^{\infty} \frac{z^{m}}{m !(m+1)^{k}}
$$

Then we have

$$
\operatorname{Lif}_{k}(\log (1+t))=\sum_{n=0}^{\infty} c_{n}^{(k)} \frac{t^{n}}{n !} .
$$

Recently there have been extensive studies in generalizing poly-Bernoulli and polyCauchy numbers. In [9], poly-Bernoulli numbers are generalized with a $q$ parameter. In [25], shifted poly-Cauchy and poly-Bernoulli numbers are defined and in [23] these numbers are further generalized with a $q$ parameter. In $[12,16]$, poly-Bernoulli and poly-Cauchy numbers and polynomials are considered by means of multiparameters.

The objective of this paper is to give further generalizations for poly-Bernoulli and poly-Cauchy numbers. These generalizations, in particular, give some symmetries for Stirling number series, and lead to a unified investigation of arithmetic and algebraic properties for poly-Bernoulli and poly-Cauchy numbers. Moreover since the multiple zeta values and the Arakawa-Kaneko zeta functions are closely related to poly-Bernoulli numbers and polynomials, inverse binomial series and Bernoulli polynomial series (see $[3,10,11,32,33]$ ), the generalizations in this paper may lead to further investigations related to various zeta functions. One of the generalizations is inspired from the polylogarithm function. Since the polylogarithm function can be derived from the Hurwitz-Lerch zeta function, poly-Bernoulli numbers are generalized by using this more general function. Similar modification leads to a generalization for poly-Cauchy numbers. The other generalizations come from the combinatorial identities (3) and (4). These formulas, which involve Stirling numbers, are reconsidered by employing weighted and degenerate weighted Stirling numbers. Numerous formulas 
such as a recurrence formula and a symmetry for generalizations of Stirling numbers, and some divisibility properties including partial congruences are also presented.

\section{Generalizations with Hurwitz-Lerch zeta function}

A general Hurwitz-Lerch zeta function $\Phi(z, s, a)$ is defined for $s \in \mathbb{C}$ when $|z|<1$, $\operatorname{Re} s>1$ when $|z|=1$ and $a \notin\{0,-1,-2, \ldots\}$ by the series ([30])

$$
\Phi(z, s, a)=\sum_{n=0}^{\infty} \frac{z^{n}}{(n+a)^{s}} .
$$

This function includes several special functions, one of which is the polylogarithm function

$$
\operatorname{Li}_{s}(z)=\sum_{n=1}^{\infty} \frac{z^{n}}{n^{s}}=z \Phi(z, s, 1) .
$$

Since

$$
\frac{\operatorname{Li}_{k}\left(1-\mathrm{e}^{-t}\right)}{1-\mathrm{e}^{-t}}=\sum_{n=0}^{\infty} B_{n}^{(k)} \frac{t^{n}}{n !}
$$

equation (5) motivates the generalization

$$
\Phi\left(1-\mathrm{e}^{-t}, s, a\right)=\sum_{n=0}^{\infty} B_{n}^{(k)}(a) \frac{t^{n}}{n !},
$$

so that $B_{n}^{(k)}(1)=B_{n}^{(k)}$. We call $B_{n}^{(k)}(a)$ the Hurwitz type poly-Bernoulli numbers. An explicit formula can be obtained by direct use of (2) and (6) as follows

Theorem 2.1 For the Hurwitz type poly-Bernoulli numbers $B_{n}^{(k)}(a)$ we have

$$
B_{n}^{(k)}(a)=(-1)^{n} \sum_{m=0}^{n} \frac{(-1)^{m} m ! S_{2}(n, m)}{(m+a)^{k}}
$$

Proof We have

$$
\begin{aligned}
\sum_{n=0}^{\infty} B_{n}^{(k)}(a) \frac{t^{n}}{n !} & =\Phi\left(1-\mathrm{e}^{-t}, k, a\right)=\sum_{m=0}^{\infty} \frac{\left(1-\mathrm{e}^{-t}\right)^{m}}{(m+a)^{k}} \\
& =\sum_{m=0}^{\infty} \frac{(-1)^{m}\left(\mathrm{e}^{-t}-1\right)^{m}}{(m+a)^{k}} \\
& =\sum_{m=0}^{\infty} \frac{(-1)^{m} m !}{(m+a)^{k}} \sum_{n=m}^{\infty} S_{2}(n, m) \frac{(-t)^{n}}{n !}
\end{aligned}
$$




$$
=\sum_{n=0}^{\infty} \sum_{m=0}^{n} \frac{(-1)^{n+m} m ! S_{2}(n, m)}{(m+a)^{k}} \frac{t^{n}}{n !}
$$

by means of standard operation on series (see, for instance [20, Equation (1.11)]). Comparison of the coefficients of $t^{n} / n$ ! in the first and last terms in this string of equalities yields (7).

One of the remarkable properties of poly-Bernoulli numbers with combinatorial interpretations is a closed formula in the case of negative upper index $k$ (for combinatorial interpretations of poly-Bernoulli numbers we refer to $[5,26])$. Such a formula for Hurwitz type poly-Bernoulli numbers may be given in terms of weighted Stirling numbers. Carlitz [8] has defined $R_{1}(n, k, x)$ and $R(n, k, x)$ by means of

$$
\frac{(1-t)^{-x}(-\log (1-t))^{k}}{k !}=\sum_{n=0}^{\infty} R_{1}(n, k, x) \frac{t^{n}}{n !},
$$

and

$$
\frac{\mathrm{e}^{x t}\left(\mathrm{e}^{t}-1\right)^{k}}{k !}=\sum_{n=0}^{\infty} R(n, k, x) \frac{t^{n}}{n !} .
$$

For convenience we call $R_{1}(n, k, x)$ and $R(n, k, x)$ the weighted Stirling numbers of the first and second kind and denote them by $S_{1}(n, k, x)$ and $S_{2}(n, k, x)$, respectively, although Carlitz used that terminology for slightly different numbers. When $x=0$ we have $S_{1}(n, k, 0)=(-1)^{n-k} S_{1}(n, k)$ and $S_{2}(n, k, 0)=S_{2}(n, k)$, the ordinary Stirling numbers.

Theorem 2.2 For the Hurwitz type poly-Bernoulli numbers $B_{n}^{(-k)}(a)$ we have

$$
B_{n}^{(-k)}(a)=\sum_{j=0}^{\min (n, k)}(j !)^{2} S_{2}(n+1, j+1) S_{2}(k, j, a) .
$$

For the proof we need two relations regarding Stirling and weighted Stirling numbers of the second kind.

Lemma 2.3 We have

$$
\sum_{k=0}^{n}\left(\begin{array}{l}
n \\
k
\end{array}\right) S_{2}(k, j)=S_{2}(n+1, j+1)=S_{2}(n, j, 1)
$$

and

$$
\sum_{n=0}^{k} a^{k-n}\left(\begin{array}{l}
k \\
n
\end{array}\right) S_{2}(n, j)=S_{2}(k, j, a) .
$$


Proof Although the first equality (9) can be found in standard texts such as [13] we give the proof here in order to show the relation to the second equation. We start with (2). Replacing $m$ with $j+1$,

$$
\frac{\left(\mathrm{e}^{t}-1\right)^{j+1}}{(j+1) !}=\sum_{n=j+1}^{\infty} S_{2}(n, j+1) \frac{t^{n}}{n !}=\sum_{n=j}^{\infty} S_{2}(n+1, j+1) \frac{t^{n+1}}{(n+1) !} .
$$

Differentiation with respect to $t$ gives

$$
\sum_{n=j}^{\infty} S_{2}(n+1, j+1) \frac{t^{n}}{n !}=\frac{\left(\mathrm{e}^{t}-1\right)^{j} \mathrm{e}^{t}}{j !} .
$$

Now,

$$
\begin{aligned}
\sum_{n=j}^{\infty} S_{2}(n+1, j+1) \frac{t^{n}}{n !} & =\sum_{n=0}^{\infty} S_{2}(n+1, j+1) \frac{t^{n}}{n !}=\frac{\left(\mathrm{e}^{t}-1\right)^{j} \mathrm{e}^{t}}{j !} \\
& =\sum_{n=0}^{\infty} S_{2}(n, j) \frac{t^{n}}{n !} \sum_{n=0}^{\infty} \frac{t^{n}}{n !}=\sum_{n=0}^{\infty} \sum_{k=0}^{n}\left(\begin{array}{l}
n \\
k
\end{array}\right) S_{2}(k, j) \frac{t^{n}}{n !}
\end{aligned}
$$

Comparing the coefficients of $t^{n} / n$ ! on both sides gives the first part of (9). The second part follows from

$$
\sum_{n=0}^{\infty} S_{2}(n+1, j+1) \frac{t^{n}}{n !}=\frac{\left(\mathrm{e}^{t}-1\right)^{j} \mathrm{e}^{t}}{j !}=\sum_{n=0}^{\infty} S_{2}(n, j, 1) \frac{t^{n}}{n !}
$$

in a somewhat more direct manner than (9). We have

$$
\begin{aligned}
\sum_{k=0}^{\infty} \sum_{n=0}^{k} a^{k-n}\left(\begin{array}{l}
k \\
n
\end{array}\right) S_{2}(n, j) \frac{t^{k}}{k !} & =\sum_{k=0}^{\infty} \sum_{n=0}^{k} \frac{a^{k-n} t^{k-n}}{(k-n) !} \frac{S_{2}(n, j) t^{n}}{n !} \\
& =\sum_{k=0}^{\infty} \frac{(a t)^{k}}{k !} \sum_{k=0}^{\infty} S_{2}(k, j) \frac{t^{k}}{k !} \\
& =\frac{\left(\mathrm{e}^{t}-1\right)^{j} \mathrm{e}^{a t}}{j !}=\sum_{k=0}^{\infty} S_{2}(k, j, a) \frac{t^{k}}{k !}
\end{aligned}
$$

Comparison of the coefficients gives the result.

Proof of Theorem 2.2 In order to prove the theorem we calculate the generating function of $B_{n}^{(-k)}(a)$ in the following form:

$$
\sum_{k=0}^{\infty} \sum_{n=0}^{\infty} B_{n}^{(-k)}(a) \frac{t^{n}}{n !} \frac{u^{k}}{k !}=\sum_{k=0}^{\infty} \sum_{m=0}^{\infty}\left(1-\mathrm{e}^{-t}\right)^{m}(m+a)^{k} \frac{u^{k}}{k !}
$$




$$
\begin{aligned}
& =\sum_{m=0}^{\infty}\left(1-\mathrm{e}^{-t}\right)^{m} \mathrm{e}^{(m+a) u}=\mathrm{e}^{a u} \sum_{m=0}^{\infty}\left(\mathrm{e}^{u}-\mathrm{e}^{u-t}\right)^{m} \\
& =\frac{\mathrm{e}^{a u}}{1-\mathrm{e}^{u}+\mathrm{e}^{u-t}}=\frac{\mathrm{e}^{t+a u}}{1-\left(\mathrm{e}^{t}-1\right)\left(\mathrm{e}^{u}-1\right)} \\
& =\sum_{j=0}^{\infty} \mathrm{e}^{t}\left(\mathrm{e}^{t}-1\right)^{j} \mathrm{e}^{a u}\left(\mathrm{e}^{u}-1\right)^{j} \\
& =\sum_{j=0}^{\infty}\left[j ! \sum_{n=0}^{\infty} \frac{t^{n}}{n !} \sum_{n=0}^{\infty} S_{2}(n, j) \frac{t^{n}}{n !}\right]\left[j ! \sum_{k=0}^{\infty} \frac{a^{k} u^{k}}{k !} \sum_{k=0}^{\infty} S_{2}(k, j) \frac{u^{k}}{k !}\right] \\
& =\sum_{j=0}^{\infty}\left[j ! \sum_{n=0}^{\infty} \sum_{k=0}^{n}\left(\begin{array}{l}
n \\
k
\end{array}\right) S_{2}(k, j) \frac{t^{n}}{n !}\right]\left[j ! \sum_{k=0}^{\infty} \sum_{n=0}^{k} a^{k-n}\left(\begin{array}{l}
k \\
n
\end{array}\right) S_{2}(n, j) \frac{u^{k}}{k !}\right] .
\end{aligned}
$$

From (9) and (10) we get

$$
\begin{aligned}
& \sum_{k=0}^{\infty} \sum_{n=0}^{\infty} B_{n}^{(-k)}(a) \frac{t^{n}}{n !} \frac{u^{k}}{k !} \\
& \quad=\sum_{j=0}^{\infty}\left[j ! \sum_{n=0}^{\infty} S_{2}(n+1, j+1) \frac{t^{n}}{n !}\right]\left[j ! \sum_{k=0}^{\infty} S_{2}(k, j, a) \frac{u^{k}}{k !}\right] \\
& =\sum_{k=0}^{\infty} \sum_{n=0}^{\infty} \sum_{j=0}^{\min (n, k)}(j !)^{2} S_{2}(n+1, j+1) S_{2}(k, j, a) \frac{t^{n}}{n !} \frac{u^{k}}{k !}
\end{aligned}
$$

Comparing the coefficients gives the result.

We note that for $a=1$, Theorem 2.2 reduces to the closed formula given by Arakawa and Kaneko [2] and by Sánchez-Peregrino [29].

Recall that the poly-Cauchy numbers $c_{n}^{(k)}$ are defined by means of the generating function

$$
\operatorname{Lif}_{k}(\log (1+t))=\sum_{n=0}^{\infty} c_{n}^{(k)} \frac{t^{n}}{n !}
$$

where $\operatorname{Lif}_{k}(z)$ is the polylogarithm factorial function given by

$$
\operatorname{Lif}_{k}(z)=\sum_{m=0}^{\infty} \frac{z^{m}}{m !(m+1)^{k}}
$$

In order to give a Hurwitz type extension of poly-Cauchy numbers we define the Hurwitz-Lerch factorial zeta function $\Phi f(z, s, a)$ by 


$$
\Phi \mathrm{f}(z, s, a)=\sum_{n=0}^{\infty} \frac{z^{n}}{n !(n+a)^{s}} .
$$

For $a=1$ and $s=k$, a natural number, we obviously have $\Phi f(z, k, 1)=\operatorname{Lif}_{k}(z)$. Thus the Hurwitz type poly-Cauchy numbers which are denoted by $c_{n}^{(k)}(a)$ may be defined by

$$
\Phi \mathrm{f}(\log (1+t), k, a)=\sum_{n=0}^{\infty} c_{n}^{(k)}(a) \frac{t^{n}}{n !} .
$$

As in the Bernoulli case an explicit formula for the Hurwitz type poly-Cauchy numbers can be obtained involving Stirling numbers of the first kind.

Theorem 2.4 For the Hurwitz type poly-Cauchy numbers $c_{n}^{(k)}(a)$ we have

$$
c_{n}^{(k)}(a)=(-1)^{n} \sum_{m=0}^{n} \frac{(-1)^{m} S_{1}(n, m)}{(m+a)^{k}} .
$$

Proof We have

$$
\begin{aligned}
\sum_{n=0}^{\infty} c_{n}^{(k)}(a) \frac{t^{n}}{n !} & =\Phi f(\log (1+t), k, a)=\sum_{m=0}^{\infty} \frac{(\log (1+t))^{m}}{m !(m+a)^{k}} \\
& =\sum_{m=0}^{\infty} \frac{1}{(m+a)^{k}} \sum_{n=m}^{\infty}(-1)^{n-m} S_{1}(n, m) \frac{t^{n}}{n !} \\
& =\sum_{n=0}^{\infty} \sum_{m=0}^{n} \frac{(-1)^{n-m} S_{1}(n, m)}{(m+a)^{k}} \frac{t^{n}}{n !}
\end{aligned}
$$

Equating the coefficients gives (11).

We remark that the Hurwitz type poly-Cauchy numbers are defined as shifted polyCauchy numbers in [25] and a generalization is given in [23].

Komatsu [22] defined the poly-Cauchy numbers of the second kind $\widehat{c}_{n}^{(k)}$ by

$$
\operatorname{Lif}_{k}(-\log (1+t))=\sum_{n=0}^{\infty} \widehat{c}_{n}^{(k)} \frac{t^{n}}{n !}
$$

Following Komatsu we define the Hurwitz type poly-Cauchy numbers of the second kind as

$$
\Phi f(-\log (1+t), k, a)=\sum_{n=0}^{\infty} \widehat{c}_{n}^{(k)}(a) \frac{t^{n}}{n !}
$$


An explicit formula for $\widehat{c}_{n}^{(k)}(a)$ is

$$
\widehat{c}_{n}^{(k)}(a)=(-1)^{n} \sum_{m=0}^{n} \frac{S_{1}(n, m)}{(m+a)^{k}}
$$

which can be shown along the lines of the proof of Theorem 2.4.

Orthogonality and inverse relations for Stirling numbers allow us to present some relations for Hurwitz type poly-Bernoulli and poly-Cauchy numbers. From the orthogonality relations $([13, \mathrm{p} .264])$

$$
\sum_{r=m}^{n}(-1)^{n-r} S_{1}(n, r) S_{2}(r, m)=\sum_{r=m}^{n}(-1)^{n-r} S_{2}(n, r) S_{1}(r, m)=\delta_{m n},
$$

$\delta_{m n}$ being the Kronecker symbol (that is, $\delta_{m n}=1$ for $m=n$ and $\delta_{m n}=0$ otherwise), inverse relations

$$
f_{n}=\sum_{m=0}^{n}(-1)^{n+m} S_{1}(n, m) g_{m} \quad \Longleftrightarrow \quad g_{n}=\sum_{m=0}^{n} S_{2}(n, m) f_{m}
$$

follow directly. Using (13) we get the following results.

Theorem 2.5 For the Hurwitz type poly-Bernoulli numbers $B_{n}^{(k)}(a)$ we have

$$
\sum_{m=0}^{n} S_{1}(n, m) B_{m}^{(k)}(a)=\frac{n !}{(n+a)^{k}}
$$

Proof This follows from (13) by taking $f_{m}=(-1)^{m} m ! /(m+a)^{k}$ and $g_{n}=$ $(-1)^{n} B_{n}^{(k)}(a)$.

Theorem 2.6 For the Hurwitz type poly-Cauchy numbers we have

$$
\sum_{m=0}^{n} S_{2}(n, m) c_{m}^{(k)}(a)=\frac{1}{(n+a)^{k}}
$$

and

$$
\sum_{m=0}^{n} S_{2}(n, m) \widehat{c}_{m}^{(k)}(a)=\frac{(-1)^{n}}{(n+a)^{k}}
$$

Proof From the definitions, (15) follows from the duality relation (13) by taking $g_{m}=1 /(m+a)^{k}$ and $f_{n}=c_{n}^{(k)}(a)$, and (16) by taking $g_{m}=(-1)^{m} /(m+a)^{k}$ and $f_{n}=\widehat{c}_{n}^{(k)}(a)$. 
Theorem 2.7 For nonnegative integer $n$ we have

$$
\begin{aligned}
& B_{n}^{(k)}(a)=\sum_{l=0}^{n} \sum_{m=0}^{n}(-1)^{m+n} m ! S_{2}(n, m) S_{2}(m, l) c_{l}^{(k)}(a), \\
& B_{n}^{(k)}(a)=\sum_{l=0}^{n} \sum_{m=0}^{n}(-1)^{m} m ! S_{2}(n, m) S_{2}(m, l) \widehat{c}_{l}^{(k)}(a), \\
& c_{n}^{(k)}(a)=\sum_{l=0}^{n} \sum_{m=0}^{n} \frac{(-1)^{m+n}}{m !} S_{1}(n, m) S_{1}(m, l) B_{l}^{(k)}(a), \\
& \widehat{c}_{n}^{(k)}(a)=\sum_{l=0}^{n} \sum_{m=0}^{n} \frac{(-1)^{n}}{m !} S_{1}(n, m) S_{1}(m, l) B_{l}^{(k)}(a) .
\end{aligned}
$$

Proof Using (15) we compute

$$
\begin{aligned}
& \sum_{l=0}^{n} \sum_{m=0}^{n}(-1)^{m+n} m ! S_{2}(n, m) S_{2}(m, l) c_{l}^{(k)}(a) \\
& \quad=\sum_{l=0}^{n} \sum_{m=l}^{n}(-1)^{m+n} m ! S_{2}(n, m) S_{2}(m, l) c_{l}^{(k)}(a) \\
& \quad=\sum_{m=0}^{n}(-1)^{m+n} m ! S_{2}(n, m) \sum_{l=0}^{m} S_{2}(m, l) c_{l}^{(k)}(a) \\
& \quad=\sum_{m=0}^{n} \frac{(-1)^{m+n} m ! S_{2}(n, m)}{(m+a)^{k}}=B_{n}^{(k)}(a)
\end{aligned}
$$

giving (17). Similarly, use of (16) gives (18). On the other hand, by (14) we get (19) and (20).

There are no closed formulas for the poly-Cauchy numbers like those for the polyBernoulli numbers in (8). Instead we have

$$
\sum_{k=0}^{\infty} \sum_{n=0}^{\infty} c_{n}^{(-k)}(a) \frac{t^{n}}{n !} \frac{u^{k}}{k !}=\mathrm{e}^{a y}(1+t)^{\mathrm{e}^{u}}
$$

and

$$
\sum_{k=0}^{\infty} \sum_{n=0}^{\infty} \widehat{c}_{n}^{(-k)}(a) \frac{t^{n}}{n !} \frac{u^{k}}{k !}=\frac{\mathrm{e}^{a u}}{(1+t)^{\mathrm{e}^{u}}}
$$




\section{Generalizations with weighted Stirling numbers}

In this section we consider generalizations of poly-Bernoulli and poly-Cauchy numbers motivated by (3) and (4). We first review some facts about weighted Stirling numbers. As mentioned in the previous section the weighted Stirling numbers of the second kind $S_{2}(n, m, x)$ are defined by

$$
\frac{\mathrm{e}^{t x}\left(\mathrm{e}^{t}-1\right)^{m}}{m !}=\sum_{n=0}^{\infty} S_{2}(n, m, x) \frac{t^{n}}{n !}
$$

Thus the weighted Stirling numbers of the second kind are in fact ordinary Stirling numbers of the second kind with an exponential factor. So the weighted and ordinary Stirling numbers of the second kind are related by

$$
S_{2}(n, m, x)=\sum_{r=0}^{n}\left(\begin{array}{l}
n \\
r
\end{array}\right) x^{r} S_{2}(n-r, m)
$$

From (21) we have $S_{2}(n, m, x)=0$ for $n<m$. Since

$$
S_{2}(n, m)=\frac{1}{m !} \sum_{j=0}^{m}(-1)^{m-j}\left(\begin{array}{c}
m \\
j
\end{array}\right) j^{n}
$$

we have

$$
S_{2}(n, m, x)=\frac{1}{m !} \sum_{j=0}^{m}(-1)^{m-j}\left(\begin{array}{c}
m \\
j
\end{array}\right)(x+j)^{n} .
$$

Multiplying (21) by $m !\left(\begin{array}{l}y \\ m\end{array}\right)$ and summing over $m$ gives

$$
\sum_{m=0}^{n} m !\left(\begin{array}{l}
y \\
m
\end{array}\right) S_{2}(n, m, x)=(y+x)^{n}
$$

Equation (23) gives the recurrence formula

$$
S_{2}(n+1, m, x)=(x+m) S_{2}(n, m, x)+S_{2}(n, m-1, x) .
$$

From (22) or (24) with $S_{2}(0,0, x)=1$ we have

$$
S_{2}(n, 0, x)=x^{n}, \quad S_{2}(n, n, x)=1 .
$$

For the weighted Stirling numbers of the first kind $S_{1}(n, m, x)$ defined in Sect. 2 by

$$
(1+t)^{-x} \frac{(\log (1+t))^{m}}{m !}=\sum_{n=0}^{\infty}(-1)^{n+m} S_{1}(n, m, x) \frac{t^{n}}{n !},
$$


we have

$$
S_{1}(n, m, x)=\sum_{r=0}^{n}\left(\begin{array}{l}
n \\
r
\end{array}\right)\langle x\rangle_{r} S_{1}(n-r, m),
$$

where $\langle x\rangle_{r}$ is the rising factorial

$$
\langle x\rangle_{r}=x(x+1) \cdots(x+r-1)
$$

with $\langle x\rangle_{0}=1$,

$$
\begin{aligned}
S_{1}(n, m, x) & =\sum_{r=0}^{n}\left(\begin{array}{c}
m+r \\
r
\end{array}\right) x^{r} S_{1}(n, m+r), \\
S_{1}(n+1, m, x) & =S_{1}(n, m-1, x)+(x+n) S_{1}(n, m, x),
\end{aligned}
$$

and

$$
S_{1}(n, 0, x)=\langle x\rangle_{n}, \quad S_{1}(n, n, x)=1 .
$$

We note from (25) that $S_{1}(n, m, x)=0$ for $n<m$.

Recall from (3) that

$$
B_{n}^{(k)}=(-1)^{n} \sum_{m=0}^{n} \frac{(-1)^{m} m ! S_{2}(n, m)}{(m+1)^{k}}
$$

In this equation we replace $S_{2}(n, m)$ by weighted Stirling number of the second kind $S_{2}(n, m, x)$. We then define weighed type poly-Bernoulli numbers by

$$
B_{n}^{(k)}(x)=(-1)^{n} \sum_{m=0}^{n} \frac{(-1)^{m} m ! S_{2}(n, m, x)}{(m+1)^{k}}
$$

From (27) we have

$$
\begin{aligned}
\sum_{n=0}^{\infty} B_{n}^{(k)}(x) \frac{t^{n}}{n !} & =\sum_{n=0}^{\infty} \sum_{m=0}^{n} \frac{(-1)^{n+m} m ! S_{2}(n, m, x)}{(m+1)^{k}} \frac{t^{n}}{n !} \\
& =\sum_{m=0}^{\infty} \frac{(-1)^{m} m !}{(m+1)^{k}} \sum_{n=0}^{\infty} S_{2}(n, m, x) \frac{(-t)^{n}}{n !} \\
& =\sum_{m=0}^{\infty} \frac{(-1)^{m} m !}{(m+1)^{k}} \frac{\mathrm{e}^{-t x}\left(\mathrm{e}^{-t}-1\right)^{m}}{m !} \\
& =\mathrm{e}^{-t x} \sum_{m=0}^{\infty} \frac{\left(1-\mathrm{e}^{-t}\right)^{m}}{(m+1)^{k}}=\mathrm{e}^{-t x} \sum_{m=1}^{\infty} \frac{\left(1-\mathrm{e}^{-t}\right)^{m-1}}{m^{k}}
\end{aligned}
$$




$$
=\frac{\mathrm{e}^{-t x}}{1-\mathrm{e}^{-t}} \sum_{m=1}^{\infty} \frac{\left(1-\mathrm{e}^{-t}\right)^{m}}{m^{k}}=\mathrm{e}^{-t x} \frac{\operatorname{Li}_{k}\left(1-\mathrm{e}^{-t}\right)}{1-\mathrm{e}^{-t}} .
$$

We remark that this kind of generalization for poly-Bernoulli numbers has recently been studied by Coppo and Candelpergher [10], Bayad and Hamahata [4], and Komatsu and Luca [24]. They called $B_{n}^{(k)}(x)$ the poly-Bernoulli polynomials. To be more precise Coppo and Candelpergher defined these polynomials as

$$
\frac{\operatorname{Li}_{k}\left(1-\mathrm{e}^{-t}\right)}{1-\mathrm{e}^{-t}} \mathrm{e}^{-x t}=\sum_{n=0}^{\infty} B_{n}^{(k)}(x) \frac{t^{n}}{n !},
$$

while Bayad and Hamahata defined them by

$$
\frac{\mathrm{Li}_{k}\left(1-\mathrm{e}^{-t}\right)}{1-\mathrm{e}^{-t}} \mathrm{e}^{x t}=\sum_{n=0}^{\infty} B_{n}^{(k)}(x) \frac{t^{n}}{n !},
$$

and Komatsu and Luca defined them by

$$
B_{n}^{(k)}(x)=(-1)^{n} \sum_{m=0}^{n}(-1)^{m} S_{2}(n, m) \sum_{i=0}^{m}\left(\begin{array}{c}
m \\
i
\end{array}\right) \frac{(-x)^{i}}{(m-i+1)^{k}}
$$

The numbers $B_{n}^{(-k)}(x)$ satisfy a closed formula like (8).

Theorem 3.1 For nonnegative integers $n$ and $k$ we have

$$
B_{n}^{(-k)}(x)=\sum_{j=0}^{\min (n, k)}(j !)^{2} S_{2}(n, j, 1-x) S_{2}(k+1, j+1) .
$$

Proof We have

$$
\begin{aligned}
& \sum_{k=0}^{\infty} \sum_{n=0}^{\infty} B_{n}^{(-k)}(x) \frac{t^{n}}{n !} \frac{u^{k}}{k !}=\sum_{k=0}^{\infty} \sum_{m=0}^{\infty} \mathrm{e}^{-x t}\left(1-\mathrm{e}^{-t}\right)^{m}(m+1)^{k} \frac{u^{k}}{k !} \\
& =\mathrm{e}^{u-x t} \sum_{m=0}^{\infty}\left(\mathrm{e}^{u}-\mathrm{e}^{u-t}\right)^{m} \\
& =\mathrm{e}^{u-x t} \frac{1}{1-\mathrm{e}^{u}+\mathrm{e}^{u-t}}=\mathrm{e}^{u+(1-x) t} \frac{1}{1-\left(\mathrm{e}^{t}-1\right)\left(\mathrm{e}^{u}-1\right)} \\
& =\sum_{j=0}^{\infty} \mathrm{e}^{(1-x) t}\left(\mathrm{e}^{t}-1\right)^{j} \mathrm{e}^{u}\left(\mathrm{e}^{u}-1\right)^{j} \\
& =\sum_{j=0}^{\infty}\left[j ! \sum_{n=0}^{\infty} S_{2}(n, j, 1-x) \frac{t^{n}}{n !}\right]\left[j ! \sum_{k=0}^{\infty} S_{2}(k, j, 1) \frac{u^{k}}{k !}\right]
\end{aligned}
$$




$$
=\sum_{k=0}^{\infty} \sum_{n=0}^{\infty} \sum_{j=0}^{\min (n, k)}(j !)^{2} S_{2}(n, j, 1-x) S_{2}(k+1, j+1) \frac{t^{n}}{n !} \frac{u^{k}}{k !},
$$

since $S_{2}(n, m, x)=0$ for $n<m$ and $S_{2}(k, j, 1)=S_{2}(k+1, j+1)$. The result now follows by comparing the coefficients.

Recall from (4) that

$$
c_{n}^{(k)}=(-1)^{n} \sum_{m=0}^{n} \frac{(-1)^{m} S_{1}(n, m)}{(m+1)^{k}}, \quad \widehat{c}_{n}^{(k)}=(-1)^{n} \sum_{m=0}^{n} \frac{S_{1}(n, m)}{(m+1)^{k}} .
$$

This last equation was given by Komatsu [22] and can be derived from (12) with $a=1$. Writing $S_{1}(n, m, x)$ instead of $S_{1}(n, m)$, we define weighted type poly-Cauchy numbers of the first kind and of the second kind by

$$
c_{n}^{(k)}(x)=(-1)^{n} \sum_{m=0}^{n} \frac{(-1)^{m} S_{1}(n, m, x)}{(m+1)^{k}}
$$

and

$$
\widehat{c}_{n}^{(k)}(x)=(-1)^{n} \sum_{m=0}^{n} \frac{S_{1}(n, m, x)}{(m+1)^{k}} .
$$

From (28) and (26) we have

$$
\begin{aligned}
c_{n}^{(k)}(x) & =(-1)^{n} \sum_{m=0}^{n} \frac{(-1)^{m}}{(m+1)^{k}} \sum_{r=0}^{n}\left(\begin{array}{c}
m+r \\
r
\end{array}\right) x^{r} S_{1}(n, m+r) \\
& =(-1)^{n} \sum_{m=r}^{n+r} \frac{(-1)^{m-r}}{(m-r+1)^{k}} \sum_{r=0}^{n}\left(\begin{array}{c}
m \\
r
\end{array}\right) x^{r} S_{1}(n, m) \\
& =(-1)^{n} \sum_{r=0}^{n} \sum_{m=r}^{n} \frac{(-1)^{m-r}}{(m-r+1)^{k}}\left(\begin{array}{c}
m \\
r
\end{array}\right) x^{r} S_{1}(n, m) \\
& =\sum_{m=0}^{n} S_{1}(n, m)(-1)^{n+m} \sum_{r=0}^{m}\left(\begin{array}{c}
m \\
r
\end{array}\right) \frac{(-x)^{r}}{(m-r+1)^{k}} .
\end{aligned}
$$

We also have

$$
\begin{aligned}
\sum_{n=0}^{\infty} c_{n}^{(k)}(x) \frac{t^{n}}{n !} & =\sum_{n=0}^{\infty} \sum_{m=0}^{n} \frac{(-1)^{n+m} S_{1}(n, m, x)}{(m+1)^{k}} \frac{t^{n}}{n !} \\
& =\sum_{m=0}^{\infty} \frac{1}{(m+1)^{k}} \sum_{n=0}^{\infty}(-1)^{n+m} S_{2}(n, m, x) \frac{t^{n}}{n !}
\end{aligned}
$$




$$
\begin{aligned}
& =\sum_{m=0}^{\infty} \frac{(1+t)^{-x}(\log (1+t))^{m}}{(m+1)^{k} m !} \\
& =(1+t)^{-x} \operatorname{Lif}_{k}(\log (1+t)) .
\end{aligned}
$$

In the view of above equations we note that Komatsu [21,22], Kamano and Komatsu [18], and Komatsu and Luca [24] have given these definitions and called them poly-Cauchy polynomials.

For the weighted type poly-Cauchy numbers of the second kind we similarly have

$$
\widehat{c}_{n}^{(k)}(x)=(-1)^{n} \sum_{m=0}^{n} S_{1}(n, m) \sum_{r=0}^{m}\left(\begin{array}{l}
m \\
r
\end{array}\right) \frac{(-x)^{r}}{(m-r+1)^{k}}
$$

and

$$
\sum_{n=0}^{\infty} \widehat{c}_{n}^{(k)}(x) \frac{t^{n}}{n !}=(1+t)^{-x} \operatorname{Lif}_{k}(-\log (1+t))
$$

In order to obtain combinatorial formulas for the weighted type poly-Bernoulli and poly-Cauchy numbers, we use the orthogonality and inverse relations for weighted Stirling numbers.

Orthogonality relations for weighted Stirling numbers have been given by Carlitz [8] as

$$
\sum_{r=m}^{n}(-1)^{n-r} S_{2}(n, r, x) S_{1}(r, m, x)=\sum_{r=m}^{n}(-1)^{r-m} S_{1}(n, r, x) S_{2}(r, m, x)=\delta_{m n} .
$$

We thus obtain the inverse relation

$$
f_{n}=\sum_{m=0}^{n}(-1)^{n+m} S_{1}(n, m, x) g_{m} \quad \Longleftrightarrow \quad g_{n}=\sum_{m=0}^{n} S_{2}(n, m, x) f_{m} .
$$

Theorem 3.2 For the weighted type poly-Bernoulli numbers $B_{n}^{(k)}(x)$ we have

$$
\sum_{m=0}^{n} S_{1}(n, m, x) B_{m}^{(k)}(x)=\frac{n !}{(n+1)^{k}} .
$$

Proof This follows from relation (29) by taking $f_{m}=(-1)^{m} m ! /(m+1)^{k}$ and $g_{n}=$ $(-1)^{n} B_{n}^{(k)}(x)$.

Theorem 3.3 For the weighted type poly-Cauchy numbers of the first and second kind we have

$$
\sum_{m=0}^{n} S_{2}(n, m, x) c_{m}^{(k)}(x)=\frac{1}{(n+1)^{k}}
$$


and

$$
\sum_{m=0}^{n} S_{1}(n, m, x) \widehat{c}_{m}^{(k)}(x)=\frac{(-1)^{n}}{(n+1)^{k}} .
$$

Proof From the definitions, (30) follows from (29) by taking $g_{m}=1 /(m+1)^{k}$ and $f_{n}=c_{n}^{(k)}(x)$, and (31) by taking $g_{m}=(-1)^{m} /(m+1)^{k}$ and $f_{n}=\widehat{c}_{n}^{(k)}(x)$.

Theorem 3.4 For nonnegative integer $n$ and any $x, y$ we have

$$
\begin{aligned}
& B_{n}^{(k)}(x)=\sum_{l=0}^{n} \sum_{m=0}^{n}(-1)^{m+n} m ! S_{2}(n, m, x) S_{2}(m, l, y) c_{l}^{(k)}(y), \\
& B_{n}^{(k)}(x)=\sum_{l=0}^{n} \sum_{m=0}^{n}(-1)^{m} m ! S_{2}(n, m, x) S_{2}(m, l, y) \widehat{c}_{l}^{(k)}(y), \\
& c_{n}^{(k)}(x)=\sum_{l=0}^{n} \sum_{m=0}^{n} \frac{(-1)^{m+n}}{m !} S_{1}(n, m, x) S_{1}(m, l, y) B_{l}^{(k)}(y), \\
& \widehat{c}_{n}^{(k)}(x)=\sum_{l=0}^{n} \sum_{m=0}^{n} \frac{(-1)^{n}}{m !} S_{1}(n, m, x) S_{1}(m, l, y) B_{l}^{(k)}(y) .
\end{aligned}
$$

Proof The definitions of $B_{n}^{(k)}(x), c_{n}^{(k)}(x)$ and $\widehat{c}_{n}^{(k)}(x)$, together with Theorems 3.2 and 3.3 give the results.

\section{Hurwitz type weighted poly-Bernoulli and poly-Cauchy numbers}

As the title indicates, we may combine the definitions of Hurwitz type and weighted type poly-Bernoulli and poly-Cauchy numbers. We define

$$
\begin{aligned}
& B_{n}^{(k)}(a, x)=(-1)^{n} \sum_{m=0}^{n} \frac{(-1)^{m} m ! S_{2}(n, m, x)}{(m+a)^{k}}, \\
& c_{n}^{(k)}(a, x)=(-1)^{n} \sum_{m=0}^{n} \frac{(-1)^{m} S_{1}(n, m, x)}{(m+a)^{k}}, \\
& \widehat{c}_{n}^{(k)}(a, x)=(-1)^{n} \sum_{m=0}^{n} \frac{S_{1}(n, m, x)}{(m+a)^{k}} .
\end{aligned}
$$

From the generating functions of Hurwitz type and weighted type numbers we obtain

$$
\begin{aligned}
& B_{n}^{(k)}(a, x)=\sum_{m=0}^{n}(-1)^{m}\left(\begin{array}{l}
n \\
m
\end{array}\right) x^{m} B_{n-m}^{(k)}(a), \\
& c_{n}^{(k)}(a, x)=\sum_{m=0}^{n}(-1)^{m}\left(\begin{array}{l}
n \\
m
\end{array}\right) x^{m} c_{n-m}^{(k)}(a),
\end{aligned}
$$




$$
\widehat{c}_{n}^{(k)}(a, x)=\sum_{m=0}^{n}(-1)^{m}\left(\begin{array}{l}
n \\
m
\end{array}\right) x^{m} \widehat{c}_{n-m}^{(k)}(a) .
$$

The generating functions of weighted Stirling numbers suggest two different recurrence formulas, namely,

$$
S_{2}(n, m, x+1)=(m+1) S_{2}(n, m+1, x)+S_{2}(n, m, x)
$$

and

$$
S_{1}(n, m, x)=S_{1}(n, m, x+1)-n S_{1}(n-1, m, x+1) .
$$

These recurrences lead to the following interesting results:

$$
\begin{aligned}
& B_{n}^{(k)}(a, x+1)+B_{n}^{(k)}(a-1, x)=B_{n}^{(k)}(a, x)+\frac{(-1)^{n} x^{n}}{(a-1)^{k}}, \quad a \neq 1, \\
& c_{n}^{(k)}(a, x+1)-n c_{n-1}^{(k)}(a, x+1)=c_{n}^{(k)}(a, x), \\
& \widehat{c}_{n}^{(k)}(a, x+1)-n \widehat{c}_{n-1}^{(k)}(a, x+1)=\widehat{c}_{n}^{(k)}(a, x) .
\end{aligned}
$$

\section{Degenerate poly-Bernoulli and poly-Cauchy numbers}

The method that extends Bernoulli and Cauchy numbers to poly-Bernoulli and polyCauchy numbers can be adapted to different kinds of number sequences. In this section we consider poly-extensions of degenerate Bernoulli and Cauchy numbers.

The degenerate Bernoulli numbers $\beta_{n}(\lambda)$ were defined by Carlitz [7] as

$$
\frac{t}{(1+\lambda t)^{\mu}-1}=\sum_{n=0}^{\infty} \beta_{n}(\lambda) \frac{t^{n}}{n !}
$$

where $\lambda \mu=1$. Note that the limiting case $\lambda=0$ gives the generating function of the ordinary Bernoulli numbers, so $\beta_{n}(0)=B_{n}$.

Following Kaneko we define the degenerate poly-Bernoulli numbers $\beta_{n}^{(k)}(\lambda)$ by

$$
\frac{\operatorname{Li}_{k}\left(1-(1-\lambda t)^{\mu}\right)}{1-(1-\lambda t)^{\mu}}=\sum_{n=0}^{\infty} \beta_{n}^{(k)}(\lambda) \frac{t^{n}}{n !} .
$$

Carlitz [7] also defined degenerate Stirling numbers of the second kind $S_{2}(n, m \mid \lambda)$ by

$$
\left((1+\lambda t)^{\mu}-1\right)^{m}=m ! \sum_{n=m}^{\infty} S_{2}(n, m \mid \lambda) \frac{t^{n}}{n !} .
$$

Equations (32) and (33) give an explicit formula for degenerate poly-Bernoulli numbers. 
Theorem 5.1 For the degenerate poly-Bernoulli numbers $\beta_{n}^{(k)}(\lambda)$ we have

$$
\beta_{n}^{(k)}(\lambda)=(-1)^{n} \sum_{m=0}^{n} \frac{(-1)^{m} m ! S_{2}(n, m \mid \lambda)}{(m+1)^{k}} .
$$

Proof We have

$$
\begin{aligned}
\sum_{n=0}^{\infty} \beta_{n}^{(k)}(\lambda) \frac{t^{n}}{n !} & =\frac{\operatorname{Li}_{k}\left(1-(1-\lambda t)^{\mu}\right)}{1-(1-\lambda t)^{\mu}} \\
& =\frac{1}{1-(1-\lambda t)^{\mu}} \sum_{m=1}^{\infty} \frac{\left(1-(1-\lambda t)^{\mu}\right)^{m}}{m^{k}} \\
& =\sum_{m=0}^{\infty} \frac{(-1)^{m}}{(m+1)^{k}}\left((1-\lambda t)^{\mu}-1\right)^{m} \\
& =\sum_{m=0}^{\infty} \frac{(-1)^{m} m !}{(m+1)^{k}} \sum_{n=m}^{\infty} S_{2}(n, m \mid \lambda) \frac{(-t)^{n}}{n !} \\
& =\sum_{n=0}^{\infty} \sum_{m=0}^{n} \frac{(-1)^{m+n} m ! S_{2}(n, m \mid \lambda)}{(m+1)^{k}} \frac{t^{n}}{n !}
\end{aligned}
$$

Comparing the coefficients gives the result.

We recall that the poly-Cauchy numbers are defined by

$$
\operatorname{Lif}_{k}(\log (1+t))=\sum_{n=0}^{\infty} c_{n}^{(k)} \frac{t^{n}}{n !}
$$

We therefore define degenerate poly-Cauchy numbers $c_{n}^{(k)}(\lambda)$ by

$$
\operatorname{Lif}_{k}\left(\frac{(1+t)^{\lambda}-1}{\lambda}\right)=\sum_{n=0}^{\infty} c_{n}^{(k)}(\lambda) \frac{t^{n}}{n !}
$$

To obtain an explicit formula for $c_{n}^{(k)}(\lambda)$ we use the degenerate Stirling numbers of the first kind $S_{1}(n, m \mid \lambda)$ which were defined by Carlitz [7] as

$$
\left(\frac{(1+t)^{\lambda}-1}{\lambda}\right)^{m}=m ! \sum_{n=m}^{\infty}(-1)^{n-m} S_{1}(n, m \mid \lambda) \frac{t^{n}}{n !}
$$

Theorem 5.2 For the degenerate poly-Cauchy numbers $c_{n}^{(k)}(\lambda)$ we have

$$
c_{n}^{(k)}(\lambda)=(-1)^{n} \sum_{m=0}^{n} \frac{(-1)^{m} S_{1}(n, m \mid \lambda)}{(m+1)^{k}} .
$$


Proof We have

$$
\begin{aligned}
\sum_{n=0}^{\infty} c_{n}^{(k)}(\lambda) \frac{t^{n}}{n !} & =\operatorname{Lif}_{k}\left(\frac{(1+t)^{\lambda}-1}{\lambda}\right)=\sum_{m=0}^{\infty} \frac{\left((1+t)^{\lambda}-1\right)^{m} / \lambda^{m}}{(m+1)^{k} m !} \\
& =\sum_{m=0}^{\infty} \frac{1}{(m+1)^{k}} \sum_{n=m}^{\infty}(-1)^{n-m} S_{1}(n, m \mid \lambda) \frac{t^{n}}{n !} \\
& =\sum_{n=0}^{\infty} \sum_{m=0}^{n} \frac{(-1)^{n-m} S_{1}(n, m \mid \lambda)}{(m+1)^{k}} \frac{t^{n}}{n !}
\end{aligned}
$$

Comparing the coefficients proves the theorem.

The degenerate Stirling numbers of the first and second kind satisfy the orthogonality relation

$$
\begin{aligned}
\sum_{r=m}^{n}(-1)^{n-r} S_{2}(n, r \mid \lambda) & S_{1}(r, m \mid \lambda) \\
& =\sum_{r=m}^{n}(-1)^{r-m} S_{1}(n, r \mid \lambda) S_{2}(r, m \mid \lambda)=\delta_{n m}
\end{aligned}
$$

([7, Equation (1.14)]). From (36) we obtain the inverse relation

$$
f_{n}=\sum_{m=0}^{n}(-1)^{n+m} S_{1}(n, m \mid \lambda) g_{m} \quad \Longleftrightarrow \quad g_{n}=\sum_{m=0}^{n} S_{2}(n, m \mid \lambda) f_{m},
$$

which provides the following results.

Theorem 5.3 For degenerate poly-Bernoulli and poly-Cauchy numbers we have

$$
\sum_{m=0}^{n} S_{1}(n, m \mid \lambda) \beta_{m}^{(k)}(\lambda)=\frac{n !}{(n+1)^{k}}
$$

and

$$
\sum_{m=0}^{n} S_{2}(n, m \mid \lambda) c_{m}^{(k)}(\lambda)=\frac{1}{(n+1)^{k}} .
$$

Proof Relation (38) follows from (37) with $f_{m}=(-1)^{m} m ! /(m+1)^{k}$ and $g_{n}=$ $(-1)^{n} \beta_{n}^{(k)}(\lambda)$, while relation (39) follows from (37) with $g_{m}=1 /(m+1)^{k}$ and $f_{n}=c_{n}^{(k)}(\lambda)$.

Theorem 5.4 For nonnegative integer $n$ and any $\lambda, \theta$ we have

$$
\beta_{n}^{(k)}(\lambda)=\sum_{l=0}^{n} \sum_{m=0}^{n}(-1)^{n+m} m ! S_{2}(n, m \mid \lambda) S_{2}(m, l \mid \theta) c_{l}^{(k)}(\theta),
$$




$$
c_{n}^{(k)}(\lambda)=\sum_{l=0}^{n} \sum_{m=0}^{n} \frac{(-1)^{n+m}}{m !} S_{1}(n, m \mid \lambda) S_{1}(m, l \mid \theta) \beta_{l}^{(k)}(\theta) .
$$

Proof For (41) we use (39) and obtain

$$
\begin{aligned}
& \sum_{l=0}^{n} \sum_{m=0}^{n} \frac{(-1)^{n+m}}{m !} S_{1}(n, m \mid \lambda) S_{1}(m, l \mid \theta) \beta_{l}^{(k)}(\theta) \\
& \quad=\sum_{l=0}^{n} \sum_{m=l}^{n} \frac{(-1)^{n+m}}{m !} S_{1}(n, m \mid \lambda) S_{1}(m, l \mid \theta) \beta_{l}^{(k)}(\theta) \\
& \quad=\sum_{m=0}^{n} \frac{(-1)^{n+m}}{m !} S_{1}(n, m \mid \lambda) \sum_{l=0}^{m} S_{1}(m, l \mid \theta) \beta_{l}^{(k)}(\theta) \\
& \quad=\sum_{m=0}^{n} \frac{(-1)^{n+m}}{m !} S_{1}(n, m \mid \lambda) \frac{m !}{(m+1)^{k}}=c_{n}^{(k)}(\lambda) .
\end{aligned}
$$

Similarly (38) gives (40).

\section{Degenerate weighted poly-Bernoulli and poly-Cauchy numbers}

We may further generalize poly-Bernoulli and poly-Cauchy numbers by means of degenerate weighted Stirling numbers. The degenerate weighted Stirling numbers of the first and second kind are defined by Howard [14] respectively as

$$
(1+t)^{\lambda-x}\left(\frac{(1+t)^{\lambda}-1}{\lambda}\right)^{m}=m ! \sum_{n=m}^{\infty}(-1)^{n-m} S_{1}(n, m, x \mid \lambda) \frac{t^{n}}{n !}
$$

and

$$
(1+\lambda t)^{\mu x}\left((1+\lambda t)^{\mu}-1\right)^{m}=m ! \sum_{n=m}^{\infty} S_{2}(n, m, x \mid \lambda) \frac{t^{n}}{n !} .
$$

We define degenerate weighted poly-Bernoulli $\beta_{n}^{(k)}(x, \lambda)$ and poly-Cauchy numbers $c_{n}^{(k)}(x, \lambda)$ by simply replacing the degenerate Stirling numbers in (34) and (35) with $S_{2}(n, m, x \mid \lambda)$ and $S_{1}(n, m, x \mid \lambda)$. Thus

$$
\beta_{n}^{(k)}(x, \lambda)=(-1)^{n} \sum_{m=0}^{n} \frac{(-1)^{m} m ! S_{2}(n, m, x \mid \lambda)}{(m+1)^{k}}
$$

are the degenerate weighted poly-Bernoulli numbers and

$$
c_{n}^{(k)}(x, \lambda)=(-1)^{n} \sum_{m=0}^{n} \frac{(-1)^{m} S_{1}(n, m, x \mid \lambda)}{(m+1)^{k}}
$$


are the degenerate weighted poly-Cauchy numbers. Generating functions for these numbers are given as follows

Theorem 6.1 We have

$$
\frac{\operatorname{Li}_{k}\left(1-(1-\lambda t)^{\mu}\right)}{1-(1-\lambda t)^{\mu}}(1-\lambda t)^{\mu x}=\sum_{n=0}^{\infty} \beta_{n}^{(k)}(x, \lambda) \frac{t^{n}}{n !}
$$

and

$$
\operatorname{Lif}_{k}\left(\frac{(1+t)^{\lambda}-1}{\lambda}\right)(1+t)^{\lambda-x}=\sum_{n=0}^{\infty} c_{n}^{(k)}(x, \lambda) \frac{t^{n}}{n !}
$$

Proof From (43) and (44) we have

$$
\begin{aligned}
\sum_{n=0}^{\infty} \beta_{n}^{(k)}(x, \lambda) \frac{t^{n}}{n !} & =\sum_{n=0}^{\infty} \sum_{m=0}^{n} \frac{(-1)^{n+m} m ! S_{2}(n, m, x \mid \lambda)}{(m+1)^{k}} \frac{t^{n}}{n !} \\
& =\sum_{m=0}^{\infty} \frac{(-1)^{m} m !}{(m+1)^{k}} \sum_{n=m}^{\infty} S_{2}(n, m, x \mid \lambda) \frac{(-t)^{n}}{n !} \\
& =\sum_{m=0}^{\infty} \frac{(-1)^{m}}{(m+1)^{k}}(1-\lambda t)^{\mu x}\left((1-\lambda t)^{\mu}-1\right)^{m} \\
& =(1-\lambda t)^{\mu x} \sum_{m=0}^{\infty} \frac{\left(1-(1-\lambda t)^{\mu}\right)^{m}}{(m+1)^{k}} \\
& =\frac{(1-\lambda t)^{\mu x}}{1-(1-\lambda t)^{\mu}} \sum_{m=1}^{\infty} \frac{\left(1-(1-\lambda t)^{\mu}\right)^{m}}{m^{k}} \\
& =\frac{\operatorname{Li}_{k}\left(1-(1-\lambda t)^{\mu}\right)}{1-(1-\lambda t)^{\mu}}(1-\lambda t)^{\mu x} .
\end{aligned}
$$

Similarly (42) and (45) yield the second result.

From Theorem 6.1 we observe that $\beta_{n}^{(k)}(0, \lambda)=\beta_{n}^{(k)}(\lambda)$ and $c_{n}^{(k)}(\lambda, \lambda)=c_{n}^{(k)}(\lambda)$.

In order to obtain relations among degenerate weighted poly-Bernoulli and polyCauchy numbers, we need an inverse relation for the degenerate weighted Stirling numbers. Hsu and Shiue [15] defined a very general class $S(n, m ; \alpha, \beta, x)$ of sequences which generalize the Stirling numbers. An exponential generating function for $S(n, m ; \alpha, \beta, x)$ is

$$
(1+\alpha t)^{x / \alpha}\left(\frac{(1+\alpha t)^{\beta / \alpha}-1}{\beta}\right)^{m}=m ! \sum_{n=0}^{\infty} S(n, m ; \alpha, \beta, x) \frac{t^{n}}{n !}
$$


We note from (46) that $S(n, m ; 1, \lambda,-x)=(-1)^{n+m} S_{1}(n, m, x+\lambda \mid \lambda)$ and $S(n, m ; \lambda, 1, x)=S_{2}(n, m, x \mid \lambda)$. They also gave the orthogonality relation

$$
\begin{aligned}
\sum_{r=m}^{n} S(n, r ; \alpha, \beta, x) & S(r, m ; \beta, \alpha,-x) \\
& =\sum_{r=m}^{n} S(n, r ; \beta, \alpha,-x) S(r, m ; \beta, \alpha, x)=\delta_{n m} .
\end{aligned}
$$

If we write $\alpha=1, \beta=\lambda$ and replace $x$ by $-x$ in (47) we obtain the orthogonality relation for the degenerate Stirling numbers as

$$
\begin{aligned}
\sum_{r=m}^{n}(-1)^{n-r} & S_{1}(n, r, x+\lambda \mid \lambda) S_{2}(r, m, x \mid \lambda) \\
& =\sum_{r=m}^{n}(-1)^{r-m} S_{2}(n, r, x \mid \lambda) S_{1}(r, m, x+\lambda \mid \lambda)=\delta_{n m} .
\end{aligned}
$$

From (48) we easily derive the inverse relation

$$
\begin{aligned}
f_{n}=\sum_{m=0}^{n}(-1)^{n+m} S_{1}(n, m, x+\lambda \mid \lambda) g_{m} & \\
& \Longleftrightarrow \quad g_{n}=\sum_{m=0}^{n} S_{2}(n, m, x \mid \lambda) f_{m} .
\end{aligned}
$$

Theorem 6.2 For degenerate weighted poly-Bernoulli and poly-Cauchy numbers we have

$$
\sum_{m=0}^{n} S_{1}(n, m, x+\lambda \mid \lambda) \beta_{m}^{(k)}(x, \lambda)=\frac{n !}{(n+1)^{k}}
$$

and

$$
\sum_{m=0}^{n} S_{2}(n, m, x \mid \lambda) c_{m}^{(k)}(x, \lambda)=\frac{1}{(n+1)^{k}} .
$$

Proof Relation (50) follows from (49) by setting $f_{m}=(-1)^{m} m ! /(m+1)^{k}$ and $g_{n}=(-1)^{n} \beta_{n}^{(k)}(x, \lambda)$. Relation (51) is obtained from (49) by taking $g_{m}=1 /(m+1)^{k}$ and $f_{n}=c_{n}^{(k)}(x, \lambda)$.

Theorem 6.3 For nonnegative integer $n$ and any values of $x, y, \lambda, \theta$ we have

$$
\begin{aligned}
& \beta_{n}^{(k)}(x, \lambda)=\sum_{l=0}^{n} \sum_{m=0}^{n}(-1)^{n+m} m ! S_{2}(n, m \mid \lambda) S_{2}(m, l, y \mid \theta) c_{l}^{(k)}(y+\theta, \theta), \\
& c_{n}^{(k)}(x, \lambda)=\sum_{l=0}^{n} \sum_{m=0}^{n} \frac{(-1)^{n+m}}{m !} S_{1}(n, m, x+\lambda \mid \lambda) S_{1}(m, l, y+\theta \mid \theta) \beta_{l}^{(k)}(y, \theta) .
\end{aligned}
$$


Proof Using Theorem 6.2,

$$
\begin{aligned}
& \sum_{l=0}^{n} \sum_{m=0}^{n}(-1)^{n+m} m ! S_{2}(n, m, x \mid \lambda) S_{2}(m, l, y \mid \theta) c_{l}^{(k)}(y+\theta, \theta) \\
& \quad=\sum_{l=0}^{n} \sum_{m=l}^{n}(-1)^{n+m} m ! S_{2}(n, m, x \mid \lambda) S_{2}(m, l, y \mid \theta) c_{l}^{(k)}(y+\theta, \theta) \\
& \quad=\sum_{m=0}^{n}(-1)^{n+m} m ! S_{2}(n, m, x \mid \lambda) \sum_{l=0}^{m} S_{2}(m, l, y \mid \theta) c_{l}^{(k)}(y+\theta, \theta) \\
& \quad=\sum_{m=0}^{n} \frac{(-1)^{n+m} m ! S_{2}(n, m, x \mid \lambda)}{(m+1)^{k}}=\beta_{n}^{(k)}(x, \lambda)
\end{aligned}
$$

giving the first part. Similarly,

$$
\begin{aligned}
& \sum_{l=0}^{n} \sum_{m=0}^{n} \frac{(-1)^{n+m}}{m !} S_{1}(n, m, x+\lambda \mid \lambda) S_{1}(m, l, y+\theta \mid \theta) \beta_{l}^{(k)}(y, \theta) \\
& \quad=\sum_{l=0}^{n} \sum_{m=l}^{n} \frac{(-1)^{n+m}}{m !} S_{1}(n, m, x+\lambda \mid \lambda) S_{1}(m, l, y+\theta \mid \theta) \beta_{l}^{(k)}(y, \theta) \\
& \quad=\sum_{m=0}^{n} \frac{(-1)^{n+m}}{m !} S_{1}(n, m, x+\lambda \mid \lambda) \sum_{l=0}^{m} S_{1}(m, l, y+\theta \mid \theta) \beta_{l}^{(k)}(y, \theta) \\
& \quad=\sum_{m=0}^{n} \frac{(-1)^{n+m}}{m !} S_{1}(n, m, x+\lambda \mid \lambda) \frac{m !}{(m+1)^{k}}=c_{n}^{(k)}(x, \lambda)
\end{aligned}
$$

gives the second part.

\section{Congruences}

The presence of Stirling numbers in explicit formulas for weighted, degenerate and degenerate weighted poly-Bernoulli and poly-Cauchy numbers enables us to discuss some divisibility properties for them. These properties give information about the denominators of these numbers. The denominators of classical Bernoulli numbers are completely determined by the von Staudt and Clausen theorem, which not only determines the denominator but also describes the fractional part of $B_{n}$. In [19] the denominators of di-Bernoulli numbers $B_{n}^{(2)}$ are completely determined while in [2] partial results for denominators of poly-Bernoulli numbers are obtained.

In this section $p$ will denote a prime number, $\mathbb{Z}_{p}$ the ring of $p$-adic integers and $\mathbb{Z}_{p}^{\times}$the multiplicative group of units in $\mathbb{Z}_{p}$. The $p$-adic valuation "ord $p$ " is defined by setting $\operatorname{ord}_{p}(x)=k$ if $x=p^{k} y$ with $y \in \mathbb{Z}_{p}^{\times}$and $\operatorname{ord}_{p}(0)=\infty$. A congruence $x \equiv y\left(\bmod m \mathbb{Z}_{p}\right)$ is equivalent to $\operatorname{ord}_{p}(x-y) \geqslant \operatorname{ord}_{p}(m)$, and if $x$ and $y$ are 
rational numbers this congruence for all primes $p$ is equivalent to the congruence $x \equiv y(\bmod m)$ given by

$$
\frac{a}{b} \equiv \frac{c}{d} \quad(\bmod m) \quad \Longleftrightarrow \quad m \mid(a d-b c),
$$

whenever $p$ does not divide $b$ and $d$.

Since degenerate weighted poly-Bernoulli and poly-Cauchy numbers are the most general cases considered so far, we present congruences for them with proofs and state the results for others as corollaries.

Theorem 7.1 Let $p$ be an odd prime number. For any $p$-adic integer $x$ and $\lambda \in \mathbb{Z}_{p}$ we have

$p^{k-1} \beta_{p}^{(k)}(x, \lambda) \equiv p^{k-1}\left(\frac{1}{2^{k}}-x\right)-\frac{[(p-1)(1-\lambda)+2 x](p-1) !}{2} \quad\left(\bmod p^{k} \mathbb{Z}_{p}\right)$

if $\lambda \in p \mathbb{Z}_{p}$ and

$$
p^{k-1} \beta_{p}^{(k)}(x, \lambda) \equiv-\frac{[(p-1)(1-\lambda)+2 x](p-1) !}{2} \quad\left(\bmod p^{k} \mathbb{Z}_{p}\right)
$$

if $\lambda \in \mathbb{Z}_{p}^{\times}$.

Proof We write (44) as

$$
\begin{aligned}
\beta_{p}^{(k)}(x, \lambda) & =(-1)^{p} \sum_{m=0}^{p} \frac{(-1)^{m} m ! S_{2}(p, m, x \mid \lambda)}{(m+1)^{k}} \\
& =-S_{2}(p, 0, x \mid \lambda)+\frac{S_{2}(p, 1, x \mid \lambda)}{2^{k}}-\frac{S_{2}(p, p-1, x \mid \lambda)(p-1) !}{p^{k}} \\
& +\frac{S_{2}(p, p, x \mid \lambda) p !}{(p+1)^{k}}-\sum_{m=2}^{p-2} \frac{(-1)^{m} m ! S_{2}(p, m, x \mid \lambda)}{(m+1)^{k}} .
\end{aligned}
$$

Since $x$ is a $p$-adic integer, $S_{2}(p, m, x \mid \lambda) \in p \mathbb{Z}_{p}$ for $1<m<p([15$, Theorem 3], [31, Equation (3.4)]). We also have $\operatorname{ord}_{p}(m !)=0$ and $\operatorname{ord}_{p}\left((m+1)^{k}\right)=0$ for $1<m<$ $p-1$. Thus the latter sum is in $p \mathbb{Z}_{p}$. For the terms $S_{2}(p, 0, x \mid \lambda)$ and $S_{2}(p, 1, x \mid \lambda)$ we use

$$
S_{2}(n, m, x \mid \lambda)=\frac{1}{m !} \sum_{r=0}^{m}(-1)^{n+r}\left(\begin{array}{l}
m \\
r
\end{array}\right)(x+r \mid \lambda)_{n}
$$

([14, Equation (4.2)]) and obtain

$$
S_{2}(p, 0, x \mid \lambda)=(x \mid \lambda)_{p}, \quad S_{2}(p, 1, x \mid \lambda)=-(x \mid \lambda)_{p}+(x+1 \mid \lambda)_{p} .
$$


From (43) we easily get $S_{2}(p, p, x \mid \lambda)=1$, and repetitive application of the recurrence formula

$$
S_{2}(n+1, m, x \mid \lambda)=(m+x-\lambda n) S_{2}(n, m, x \mid \lambda)+S_{2}(n, m-1, x \mid \lambda)
$$

([14, Equation(4.11)]) gives

$$
S_{2}(p, p-1, x \mid \lambda)=\left(\begin{array}{l}
p \\
2
\end{array}\right)(1-\lambda)+p x .
$$

Thus we have

$$
\begin{aligned}
\beta_{p}^{(k)}(x, \lambda) \equiv-(x \mid \lambda)_{p} & +\frac{(x+1 \mid \lambda)_{p}-(x \mid \lambda)_{p}}{2^{k}} \\
& -\frac{\left[\left(\begin{array}{c}
p \\
2
\end{array}\right)(1-\lambda)+p x\right](p-1) !}{p^{k}}\left(\bmod p \mathbb{Z}_{p}\right)
\end{aligned}
$$

since $p ! \in p \mathbb{Z}_{p}$. Now, since

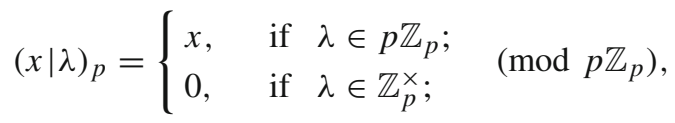

we have

$$
\begin{aligned}
p^{k-1} \beta_{p}^{(k)}(x, \lambda) \equiv p^{k-1} & \left(\frac{1}{2^{k}}-x\right) \\
& -\frac{[(p-1)(1-\lambda)+2 x](p-1) !}{2} \quad\left(\bmod p^{k} \mathbb{Z}_{p}\right)
\end{aligned}
$$

if $\lambda \in p \mathbb{Z}_{p}$ and

$$
p^{k-1} \beta_{p}^{(k)}(x, \lambda) \equiv-\frac{[(p-1)(1-\lambda)+2 x](p-1) !}{2} \quad\left(\bmod p^{k} \mathbb{Z}_{p}\right)
$$

if $\lambda \in \mathbb{Z}_{p}^{\times}$.

For $x=0$ we have the following result for degenerate poly-Bernoulli numbers.

Corollary 7.2 For an odd prime $p$ and $\lambda \in \mathbb{Z}_{p}$ we have

$$
p^{k-1} \beta_{p}^{(k)}(\lambda) \equiv \frac{p^{k-1}}{2^{k}}+\frac{(p-1)(\lambda-1)(p-1) !}{2}\left(\bmod p^{k} \mathbb{Z}_{p}\right)
$$

if $\lambda \in p \mathbb{Z}_{p}$ and

$$
p^{k-1} \beta_{p}^{(k)}(\lambda) \equiv \frac{(p-1)(\lambda-1)(p-1) !}{2} \quad\left(\bmod p^{k} \mathbb{Z}_{p}\right)
$$


if $\lambda \in \mathbb{Z}_{p}^{\times}$.

Similarly, if we let $\lambda=0$ in Theorem 7.1 we obtain

Corollary 7.3 For an odd prime $p$ and any $p$-adic integer $x$ we have

$$
p^{k-1} B_{p}^{(k)}(x) \equiv \frac{p^{k-1}}{2^{k}}-\frac{(p-1+2 x)(p-1) !}{2}\left(\bmod p^{k} \mathbb{Z}_{p}\right) .
$$

We note that similar congruences for poly-Bernoulli numbers were given in [2] and [19].

The following result gives a divisibility property for degenerate weighted polyCauchy numbers of the first kind.

Theorem 7.4 Let $p$ be an odd prime number. For any $p$-adic integer $x$ and $\lambda \in \mathbb{Z}_{p}^{\times}$ we have

$$
p^{k-1} c_{p}^{(k)}(x, \lambda) \equiv p^{k-1}-\frac{(p-1)(\lambda-1)+2 x-2 \lambda}{2} \quad\left(\bmod p^{k} \mathbb{Z}_{p}\right) .
$$

Proof Consider the defining equation

$$
c_{p}^{(k)}(x, \lambda)=(-1)^{p} \sum_{m=0}^{p} \frac{(-1)^{m} S_{1}(p, m, x \mid \lambda)}{(m+1)^{k}} .
$$

Since $\lambda \in \mathbb{Z}_{p}^{\times}$and $\lambda-x \in \mathbb{Z}_{p}$ we have $S_{1}(p, m, x \mid \lambda) \in p \mathbb{Z}_{p}$ for $1<m<p([15$, Theorem 3], [31, Equation (3.4)]). Thus we get

$$
\begin{aligned}
c_{p}^{(k)}(x, \lambda) \equiv-S_{1}(p, 0, x \mid \lambda)+\frac{S_{1}(p, 1, x \mid \lambda)}{2^{k}} & -\frac{S_{1}(p, p-1, x \mid \lambda)}{p^{k}} \\
& +\frac{S_{1}(p, p, x \mid \lambda)}{(p+1)^{k}}\left(\bmod p \mathbb{Z}_{p}\right) .
\end{aligned}
$$

From the equation

$$
\begin{aligned}
& \lambda^{m} m ! S_{1}(n, m, x \mid \lambda) \\
& \quad=\sum_{r=0}^{m}(-1)^{n+r}\left(\begin{array}{c}
m \\
r
\end{array}\right)(\lambda r+\lambda-x)(\lambda r+\lambda-x-1) \cdots(\lambda r+\lambda-x-n+1)
\end{aligned}
$$

([14, Equation(4.1)]) we obtain

$$
S_{1}(p, 0, x \mid \lambda)=(x-\lambda)_{p}, \quad S_{1}(p, 1, x \mid \lambda)=\frac{(2 \lambda-x)_{p}-(\lambda-x)_{p}}{\lambda} .
$$

From (42) we have $S_{1}(p, p, x \mid \lambda)=1$ and

$$
S_{1}(n, m, x \mid \lambda)=\sum_{r=0}^{n}\left(\begin{array}{l}
n \\
r
\end{array}\right)(x-\lambda)(x-\lambda+1) \cdots(x-\lambda+r-1) S_{1}(n-r, m \mid \lambda)
$$


$([14$, p. 51]) gives

$$
S_{1}(p, p-1, x \mid \lambda)=\left(\begin{array}{l}
p \\
2
\end{array}\right)(1-\lambda)+p x-p \lambda
$$

since $S_{1}(p, p-1 \mid \lambda)=\left(\begin{array}{l}p \\ 2\end{array}\right)(1-\lambda)$. Thus we have

$$
\begin{aligned}
c_{p}^{(k)}(x, \lambda) \equiv 1-(x-\lambda)_{p} & +\frac{(2 \lambda-x)_{p}-(\lambda-x)_{p}}{\lambda 2^{k}} \\
& +\frac{p(x-\lambda)\left(\begin{array}{l}
p \\
2
\end{array}\right)(1-\lambda)}{p^{k}} \quad\left(\bmod p \mathbb{Z}_{p}\right)
\end{aligned}
$$

Now, since for any $z \in \mathbb{Z}_{p}$ we have $(z)_{p} \in p \mathbb{Z}_{p}$, we see that $p^{k-1}(x-\lambda)_{p}$ and $p^{k-1}(2 \lambda-x)_{p}$ are zero modulo $p^{k}$. Therefore we obtain

$$
p^{k-1} c_{p}^{(k)}(x, \lambda) \equiv p^{k-1}+\frac{(p-1)(1-\lambda)+2 \lambda-2 x}{2} \quad\left(\bmod p^{k} \mathbb{Z}_{p}\right)
$$

which is the result.

For $\lambda=0$ we have the following result.

Corollary 7.5 For an odd prime $p$ and any p-adic integer $x$ we have

$$
p^{k-1} c_{p}^{(k)}(x) \equiv p^{k-1}-\frac{p-1+2 x}{2} \quad\left(\bmod p^{k} \mathbb{Z}_{p}\right)
$$

The next result states a divisibility property for $c_{n}^{(k)}(\lambda)$, which follows from Theorem 7.4 with $x=\lambda$.

Corollary 7.6 Let $p$ be an odd prime. Then

$$
p^{k-1} c_{p}^{(k)}(\lambda) \equiv p^{k-1}+\frac{(p-1)(\lambda-1)}{2} \quad\left(\bmod p^{k} \mathbb{Z}_{p}\right)
$$

if $\lambda \in \mathbb{Z}_{p}^{\times}$and

$$
p^{k-1} c_{p}^{(k)}(\lambda) \equiv \frac{(p-1)(\lambda-1)}{2} \quad\left(\bmod p^{k-1} \mathbb{Z}_{p}\right)
$$

if $\lambda \in p \mathbb{Z}_{p}$.

For the second result we note that if $\lambda \in p \mathbb{Z}_{p}$ then $S_{1}(p, m \mid \lambda)$ is in $\mathbb{Z}_{p}$ not in $p \mathbb{Z}_{p}$. Thus when $\lambda \in p \mathbb{Z}_{p}$ we conclude that

$$
p^{k-1} c_{p}^{(k)}(\lambda) \equiv \frac{(p-1)(\lambda-1)}{2} \quad\left(\bmod p^{k-1} \mathbb{Z}_{p}\right)
$$


Acknowledgments The authors would like to thank referees for their many valuable comments and suggestions.

\section{References}

1. Arakawa, T., Ibukiyama, T., Kaneko, M.: Bernoulli Numbers and Zeta Functions. Springer Monographs in Mathematics. Springer, Tokyo (2014)

2. Arakawa, T., Kaneko, M.: On poly-Bernoulli numbers. Comment. Math. Univ. St. Paul 48(2), 159-167 (1999)

3. Arakawa, T., Kaneko, M.: Multiple zeta values, poly-Bernoulli numbers, and related zeta functions. Nagoya Math. J. 153, 189-209 (1999)

4. Bayad, A., Hamahata, Y.: Polylogarithms and poly-Bernoulli polynomials. Kyushu J. Math. 65(1), 15-24 (2011)

5. Brewbaker, C.: A combinatorial interpretation of the poly-Bernoulli numbres and two Fermat analogues. Integers 8(1), \# A2 (2008)

6. Carlitz, L.: A note on Bernoulli and Euler polynomials of the second kind. Scripta Math. 25, 323-330 (1961)

7. Carlitz, L.: Degenerate Stirling. Bernoulli and Eulerian numbers. Util. Math. 15, 51-88 (1979)

8. Carlitz, L.: Weighted Stirling numbers of the first and second kind-I. Fibonacci Quart. 18(2), 147-162 (1980)

9. Cenkci, M., Komatsu, T.: Poly-Bernoulli numbers and polynomials with a $q$ parameter. J. Number Theory 152, 38-54 (2015)

10. Coppo, M.-A., Candelpergher, B.: The Arakawa-Kaneko zeta functions. Ramanujan J. 22(2), 153-162 (2010)

11. Coppo, M.-A., Candelpergher, B.: Inverse binomial series and values of Arakawa-Kaneko zeta functions. J. Number Theory 150, 98-119 (2015)

12. El-Desouky, B.S., Gomaa, R.S.: Multiparameter poly-Cauchy and poly-Bernoulli numbers and polynomials (2014). arXiv:1410.5300

13. Graham, R.L., Knuth, D.E., Patashnik, O.: Concrete Mathematics, 2nd edn. Addison-Wesley, Reading (1994)

14. Howard, F.T.: Degenerate weighted Stirling numbers. Discrete Math. 57(1-2), 45-58 (1985)

15. Hsu, L.C., Shiue, P.J.-S.: A unified approach to generalized Stirling numbers. Adv. Appl. Math. 20(3), 366-384 (1998)

16. Jolany, H., Corcino, R.B.: Explicit formula for generalization of poly-Bernoulli numbers and polynomials with $a, b, c$ parameters (2011). arXiv:1109.1387

17. Jordan, C.: Calculus of Finite Differences, 2nd edn. Chelsea, New York (1950)

18. Kamano, K., Komatsu, T.: Poly-Cauchy polynomials. Mosc. J. Comb. Number Theory 3(2), 61-87 (2013)

19. Kaneko, M.: Poly-Bernoulli numbers. J. Théor. Nombres Bordeaux 9(1), 221-228 (1997)

20. Knuth, D.E.: Two notes on notation. Amer. Math. Monthly 99(5), 403-422 (1992)

21. Komatsu, T.: Poly-Cauchy numbers with a $q$ parameter. Ramanujan J. 31(3), 353-371 (2013)

22. Komatsu, T.: Poly-Cauchy numbers. Kyushu J. Math. 67(1), 143-153 (2013)

23. Komatsu, T., Laohakosol, V., Liptai, K.: A generalization of poly-Cauchy numbers and their properties. Abstr. Appl. Anal. \# 179841 (2013)

24. Komatsu, T., Luca, F.: Some relationships between poly-Cauchy numbers and poly-Bernoulli numbers. Ann. Math. Inform. 41, 99-105 (2013)

25. Komatsu, T., Szalay, L.: Shifted poly-Cauchy numbers. Lithuanian Math. J. 54(2), 166-181 (2014)

26. Launois, S.: Combinatorics of $\mathscr{H}$-primes in quantum matrices. J. Algebra 309(1), 139-167 (2007)

27. Merlini, D., Sprugnoli, R., Verri, M.C.: The Cauchy numbers. Discrete Math. 306(16), 1906-1920 (2006)

28. Nörlund, N.E.: Vorlesungen über Differenzenrechnung. Chelsea, New York (1954)

29. Sánchez-Peregrino, R.: Closed formula for poly-Bernoulli numbers. Fibonacci Quart. 40(4), 362-364 (2002)

30. Srivastava, H.M., Choi, J.: Series Associated with the Zeta and Related Functions. Kluwer, Dordrecht (2001)

31. Young, P.T.: Congruences for degenerate number sequences. Discrete Math. 270(1-3), 279-289 (2003) 
32. Young, P.T.: Symmetries of Bernoulli polynomial series and Arakawa-Kaneko zeta functions. J. Number Theory 143, 142-161 (2014)

33. Young, P.T.: The $p$-adic Arakawa-Kaneko zeta functions and $p$-adic Lerch transcendent. J. Number Theory 155, 13-35 (2015) 